\title{
COMUNICAÇÃO
}

\section{INTENSIDADE DO OÍDIO Microsphaera diffusa CKE. \& PK. EM CULTIVARES DE SOJA [Glycine max (L.) Merr.] SOB DIFERENTES TEMPERATURAS E PERÍODOS DE MOLHAMENTO FOLIAR}

\author{
Effects of temperature and leaf wetness period in powdery mildew \\ Microsphaera diffusa Cke. \& Pk. intensity in soybean [Glycine max (L.) Merr.] cultivars
}

\author{
Marcelo de Carvalho Alves', Edson Ampélio Pozza², \\ João de Cássia do Bomfim Costa ${ }^{3}$, Josimar Batista Ferreira ${ }^{4}$, Dejânia Vieira de Araújo ${ }^{5}$
}

\begin{abstract}
RESUMO
Objetivou-se, neste trabalho, verificar o efeito da temperatura e do período de molhamento foliar no progresso da severidade do oídio Microsphaera diffusa Cke. \& Pk., nas cultivares de soja Conquista e Suprema [Glycine max (L.) Merr.]. As plantas foram inoculadas em casa-de-vegetação por agitação de plantas doentes sobre plantas sadias no estádio vegetativo $\mathrm{V}_{3}$. Em seguida foram colocadas, ao acaso, em câmaras de crescimento vegetal, nas temperaturas de $15,20,25$ e $30^{\circ} \mathrm{C}$ e períodos de molhamento foliar de 0 , $6,12,18$ e $24 \mathrm{~h}$. Dados da severidade foram utilizados para avaliar o progresso da doença e integrados ao longo do tempo por meio da área abaixo da curva de progresso da severidade (AACPS). Modelos de regressão não-linear foram ajustados para AACPS. O progresso da intensidade do oídio (AACPS) nas cultivares Conquista e Suprema foi favorecido por temperaturas próximas a $23^{\circ} \mathrm{C}$ e $24^{\circ} \mathrm{C}$, respectivamente. Períodos de molhamento foliar de $8 \mathrm{~h}$ proporcionaram o progresso máximo da severidade da doença na cultivar Conquista sob temperatura de $23^{\circ} \mathrm{C}$. Temperaturas próximas de $30^{\circ} \mathrm{C}$ e de $15^{\circ} \mathrm{C}$ reduziram a intensidade da doença.
\end{abstract}

Termos para indexação: Severidade, epidemiologia, doença.

\section{ABSTRACT}

In this study the effects of temperature and leaf wetness period in the severity progress of Powdery Mildew Cke. \& Pk. in soybean cultivars Conquista and Suprema [Glycine max (L.) Merr.] were evaluated. Plants at the V3 stage were inoculated in greenhouse. Subsequently, they were conditioned into growth chambers at temperatures of $15,20,25$ and $30^{\circ} \mathrm{C}$ and leaf wetness periods of $0,6,12,18$ and 24 hours. Severity data were integrated in time by the disease progress curve for severity (AUDPCS). Nonlinear regression models were adjusted for the disease intensity. Temperatures near $23^{\circ} \mathrm{C}$ and $24^{\circ} \mathrm{C}$ favored the powdery mildew intensity progress (AUDPCS) in Conquista and Suprema cultivars, respectively. Leaf wetness period of $8 \mathrm{~h}$ allowed the maximum progress of the disease in Conquista at temperatures of $23^{\circ} \mathrm{C}$. Temperatures near $30^{\circ} \mathrm{C}$ and $15^{\circ} \mathrm{C}$ reduced powdery mildew intensity.

Index terms: Severity, epidemiology, disease.

(Recebido em 2 de julho de 2007 e aprovado em 17 de junho de 2008)

A soja [Glycine $\max ($ L.) Merr.] é cultivada em diversas regiões tropicais e subtropicais do mundo. Estados Unidos (EUA) e Brasil são os maiores produtores e exportadores mundiais dessa oleaginosa (AGRIANUAL, 2007; MIYASAKA \& MEDINA, 1981).

Apesar da elevada produção e exportação brasileira de soja, muitos fatores têm afetado a qualidade ou quantidade da produção dessa cultura, tais como epidemias de doenças, causando redução em retorno financeiro por unidade de área. Entre as doenças, encontra-se o oídio da soja, cujo agente etiológico é Microsphaera diffusa Cke. \& Pk., que por razões ainda não esclarecidas, repentinamente, passou a causar danos significativos (SARTORATO \& YORINORI, 2001). De acordo com

\footnotetext{
'Engenheiro Agrônomo, Pós-Doutor em Máquinas e Automação Agrícola, Pesquisador -Faculdade de Agronomia e Medicina Veterinária/FAMEV Departamento de Solos e Engenharia Rural/DSER - Universidade Federal de Mato Grosso/UFMT - Avenida Fernando Corrêa da Costa, s/n - Coxipó 78060-900 - Cuiabá, MT - mdecalves@ufmt.br

${ }^{2}$ Doutor em Fitopatologia, Professor - Departamento de Fitopatologia/DFP - Universidade Federal de Lavras/UFLA - Cx. P. 3037 - $37200-000$ - Lavras, MG - eapozza@ufla.br

${ }^{3}$ Engenheiro Agrônomo, Doutor em Fitopatologia - Centro de Pesquisa do Cacau/CEPEC - Comissão Executiva do Plano da Lavoura Cacaueira/ CEPLAC - Km 22, Rodovia Ilhéus/Itabuna - Cx. P. 07 - 45653-919 - Itabuna, BA - jcbcosta@uol.com.br

${ }^{4}$ Engenheiro Agrônomo, Doutor em Fitopatologia - Centro Multidisciplinar;CMULT - Universidade Federal do Acre/UFAC - Campus Floresta, Estrada Canela Fina - Km 12, Gleba Formoso, Lote 245 - Colônia São Francisco - 69980-000 - Cruzeiro do Sul, AC - josimarferreira@gmail com

${ }^{5}$ Engenheira Agrônoma, Doutora em Fitopatologia - Departamento de Agronomia/DA - Universidade do Estado de Mato Grosso/UNEMAT - Campus Universitário de Tangará da Serra - Cx. P. 287 - Jardim Aeroporto - 78300-000 - Tangará da Serra, MT - dejania@terra.com.br
} 
Yorinori \& Hiromoto (1998), lavouras amplamente atingidas pela doença, apresentaram reduções estimadas entre $30 \mathrm{e}$ $40 \%$ do rendimento, na mesma ordem de grandeza que as relatadas no exterior por Dunleavy (1978) e Philips (1984). A suscetibilidade de cultivares e a influência do clima favoreceram epidemias com altas taxas de progresso da doença, em anos sucessivos, no Brasil. A falta de resistência, na maioria das cultivares, exigiu o controle químico, principalmente no Sul do país e nas chapadas altas dos Cerrados (SARTORATO \& YORINORI, 2001).

Em relação às alterações fisiológicas no hospedeiro, Mignucci \& Boyer (1979) estudando a inibição da fotossíntese e a transpiração de soja infectada por oídio, constataram menor fotossíntese e transpiração com o aumento da infecção. Com $82 \%$ da área foliar infectada, mais da metade da atividade fotossintética da folha havia sido perdida e a transpiração baixou para $36 \%$ em relação à testemunha, como resultado da alteração direta na atividade metabólica induzida pelo patógeno.

Reações de diferentes variedades de soja ao oídio e o efeito de variáveis ambientais no progresso da doença já foram relatadas (ARNY et al., 1975; BUZZELL \& HAAS, 1975; GRAU \& LAURENCE, 1975; JOHNSON \& PHILLIPS, 1961;LOHNES \& BERNARD, 1992; LOHNES \& NICKELL, 1994; MIGNUCCI et al., 1977; MIGNUCCI \& BOYER, 1979; MIGNUCCI \& LIM, 1980). De acordo com Bedendo (1995), no Brasil, os oídios podem ocorrer em regiões úmidas e de clima frio, entretanto são favorecidos por ambientes secos e quentes $\left(20-25^{\circ} \mathrm{C}\right)$. Segundo o mesmo autor, os conídios não germinam quando forma-se um filme de água na superfície foliar, porém, umidade relativa próxima a $95 \%$ é necessária para germinação.

Mignucci et al. (1977) relataram temperaturas de $18^{\circ} \mathrm{C}$ como favoráveis ao progresso do oídio em cultivares suscetíveis e sob temperaturas de $30^{\circ} \mathrm{C}$ o progresso da doença foi inibido. Grau \& Laurence (1975) também observaram menor severidade da doença em temperatura de $30^{\circ}$ C. Segundo Sartorato \& Yorinori (2001) não há informações precisas sobre os efeitos da umidade relativa, do molhamento foliar, da precipitação pluvial, da radiação solar ou de outros fatores do ambiente no progresso do oídio da soja.

Diante disso, objetivou-se, neste trabalho, avaliar a intensidade do oídio da soja sob diferentes temperaturas e períodos de molhamento foliar, nas cultivares Conquista e Suprema, bem como comparar as cultivares com relação a essas variáveis.

Sementes de soja, das cultivares Conquista (MG/ BR 46) e Suprema foram semeadas em vaso contendo $5 \mathrm{~kg}$ da mistura solo, areia e matéria orgânica (esterco bovino) na proporção 2:1:0,5 em casa-de-vegetação. Foi realizado desbaste 15 dias após o plantio deixando-se duas plantas por vaso, constituindo a unidade experimental. As plantas foram mantidas em casa-de-vegetação até o estádio $\mathrm{V}_{3}$, de acordo com a escala fenológica da soja proposta por Ritchie et al. (1982). Nesse mesmo período, procedeu-se à inoculação de $M$. diffusa agitando plantas de soja doentes sobre sadias as quais foram posteriormente dispostas ao acaso próximo às plantas doentes (DEMSKI \& PHILLIPS, 1974). Em seguida, as plantas foram transferidas para câmaras de crescimento vegetal e arranjadas em delineamento de blocos ao acaso, esquema fatorial 4 x 5 com 3 repetições, sendo quatro temperaturas $(15,20,25$ e $\left.30^{\circ} \mathrm{C}\right)$ e cinco períodos de molhamento foliar $(0,6,12,18$ e 24 horas). Para obter os diferentes períodos de molhamento foliar as plantas recém-pulverizadas foram mantidas em câmara úmida, com sacos de plástico transparentes, pelo período de cada tratamento. No tratamento de $0 \mathrm{~h}$ de molhamento, as plantas foram levadas sem câmara úmida para as câmaras de crescimento. As irrigações foram realizadas regando-se água diretamente no colo das plantas.

Foram realizadas quatro avaliações de severidade a cada cinco dias após o início do experimento. A severidade foi avaliada no folíolo central de todos os trifólios de cada planta aos 9, 11, 13 e 15 dias após a inoculação, utilizandose a escala de notas publicada em Sartorato \& Yorinori (2001), sendo nota $1=1 \%$ da área foliar afetada; nota $2=$ $5 \%$; nota $3=10 \% ;$ nota $4=25 \%$; nota $5=50 \%$; nota $6=$ $100 \%$.

Os dados de intensidade foram integrados usandose a área abaixo da curva de progresso da severidade (AACPS) da doença ao longo do tempo, de acordo com Campbell \& Madden (1990).

$\mathrm{Na}$ análise de variância para AACPS empregou-se o esquema fatorial entre temperatura e molhamento foliar. As variáveis significativas no teste $\mathrm{F}$ foram submetidas à análise de regressão não linear e ajuste de modelos (LEITE \& AMORIM, 2002; REIS et al., 2004). No caso da interação ser significativa modelou-se o efeito combinado da temperatura e da duração do molhamento foliar na intensidade da doença (REIS et al., 2004).

Os sintomas do oídio avaliados aos 9, 11, 13 e 15 dias após a inoculação, foram caracterizados por clorose, ilhas verdes, manchas ferruginosas, e combinação desses sintomas nas cultivares Conquista e Suprema. Todavia, a característica mais marcante avaliada foi à presença de sinais do fungo, estrutura branca e pulverulenta sobre a superfície das folhas infectadas. Essa sintomatologia está de acordo com as descritas por Sartorato \& Yorinori (2001), Tanaka et al. (1993), Yorinori et al. (1993) e Yorinori \& 
Hiromoto (1998). Segundo Sartorato \& Yorinori (2001) e Yorinori et al. (1998), pode também haver variações na sintomatologia de oídio em função de variações climáticas, variabilidade genética entre populações de $M$. diffusa, resistência genética de cultivares, estádio de desenvolvimento da planta e práticas agronômicas adotadas.

Houve interação significativa, no teste $\mathrm{F}$ entre a temperatura e o molhamento foliar para a AACPS na cultivar Conquista $(\mathrm{P}=0,0242)$ e da temperatura nas cultivares Conquista $(\mathrm{P}<0,0001)$ e Suprema $(\mathrm{P}<0,0001)$. Assim, foram ajustados modelos de regressão não-linear para a AACPS do oídio da soja a fim de descrever o processo monocíclico da epidemia com base nas variáveis analisadas. Com relação à temperatura, houve maior intensidade da doença em temperaturas próximas a $23^{\circ} \mathrm{C}$ para a cultivar Conquista e a $24{ }^{\circ} \mathrm{C}$ para a cultivar Suprema. Temperaturas próximas a 15 e a $30^{\circ} \mathrm{C}$ não foram favoráveis ao progresso do oídio em ambas as cultivares (Figura 1).

Da mesma forma Leath \& Carroll (1982) em estudo sobre o oídio da soja em 38 cultivares, observaram maior suscetibilidade das cultivares Ware, Falcon, AP350, V76438, Emerald, AgDSR232, AgDSR532, Md71-583, bem como menor e maior progresso da doença ( $M$. diffusa), na localidade de Georgetown, sob condições de temperatura de $29,6^{\circ} \mathrm{C}$ e de $23,2^{\circ} \mathrm{C}$, respectivamente. No entanto, Mignucci et al. (1977) encontraram nas temperaturas de $18^{\circ} \mathrm{C}$, em casa-de-vegetação, maior progresso do oídio nas cultivares Flambeau, Norchief, Chippewa 64, Corsoy, Harosoy 63, Wells, cultivadas nos EUA e Porto Rico. As plântulas foram submetidas a temperaturas de 18,24 e $30^{\circ} \mathrm{C}$, por 14 horas, com alternância de 10 horas de temperatura a $20^{\circ} \mathrm{C}$, de modo a simular temperaturas diurnas e noturnas. Em estudos posteriores, dos mesmos autores, após a inoculação de $M$. diffusa em casa-de-vegetação, na cultivar Harosoy, as plantas doentes foram mantidas em câmara de crescimento, sob temperatura diurna de $26 \pm 2^{\circ} \mathrm{C}$ e noturna de $21 \pm 2^{\circ} \mathrm{C}$ (MIGNUCCI \& CHAMBERLAIN, 1978) e sob $25 \pm 0,25^{\circ} \mathrm{C}$ (MIGNUCCI \& BOYER, 1979). Porém, tanto Leath \& Carroll (1982), Mignucci (1989) e Mignucci et al. (1977), concordaram que temperaturas próximas a $30^{\circ} \mathrm{C}$ não são favoráveis ao progresso da doença, de forma semelhante à observada nas cultivares avaliadas neste estudo. Em outro patossistema, o oídio da videira (Uncinula necator (Schw.) Burr.), a temperatura ótima para o crescimento do fungo foi de $25^{\circ} \mathrm{C}$, sendo que na faixa térmica entre 21 a $30^{\circ} \mathrm{C}$ houve germinação de esporos e maior crescimento micelial. Sob temperaturas acima de $33^{\circ} \mathrm{C}$ houve morte de esporos e de colônias (REIS, 2004; THOMAS et al., 1994).

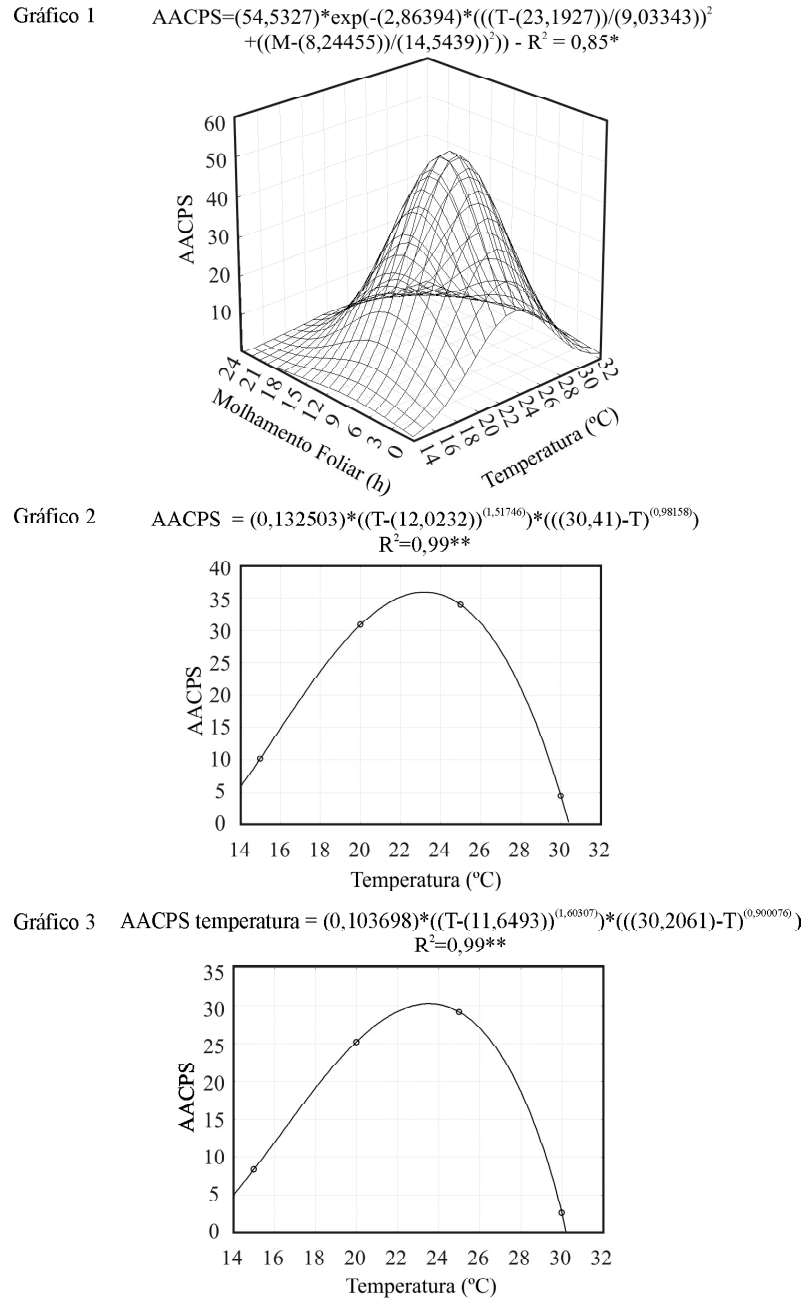

Figura 1 - Regressão não-linear do progresso da severidade (AACPS) do oídio da soja na cultivar Conquista, em função da interação entre temperatura e período de molhamento foliar (Gráfico 1) e da AACPS nas cultivares Conquista (Gráfico 2) e Suprema (Gráfico 3) em função da temperatura.

Com relação ao molhamento foliar, ocorreram sinais da doença desde a sua ausência com crescimento da AACPD até 8 horas, com o máximo de temperatura de 23 ${ }^{\circ} \mathrm{C}$, o que indica a necessidade de água para ocorrer a germinação e a infecção do fungo. A partir desse ponto, a AACPD reduziu. Existem poucas informações na literatura sobre os efeitos do molhamento foliar no oídio da soja (SARTORATO \& YORINORI, 2001), no entanto, segundo Bedendo (1995), essa doença pode ocorrer em regiões úmidas, mas é favorecida por ambientes secos. Mignucci (1989) relatou, que a baixa umidade relativa do ar é altamente favorável ao progresso do oídio da soja, entretanto não se 
apresentaram valores para descrever precisamente quanto. Da mesma forma, Brodie \& Neufeld (1942) estudando o desenvolvimento de estruturas conidiais de Erysiphe polygoni DC., constataram germinação de conídios em umidade relativa do ar variando de $0-100 \%$, enquanto Mattiazzi (2003), estudando o efeito do oídio na produção da soja, observou maior progresso sob umidade relativa de $80 \%$. Sendo assim, a umidade relativa das câmaras de crescimento vegetal, com média de $50 \%$, pode ter proporcionado a germinação de conídios, mesmo nos tratamentos com ausência de molhamento foliar.

Portanto, como os resultados sobre o efeito do molhamento foliar no progresso do oídio ( $M$. diffusa) são contraditórios e estudos sobre o efeito da interação entre a temperatura e duração de molhamento foliar no progresso da doença ainda não haviam sido avaliados, os presentes resultados apresentam-se com potencial perspectiva de utilização em estudos sobre os efeitos do clima no progresso do oídio da soja em cultivares brasileiras.

\section{REFERÊNCIAS BIBLIOGRÁFICAS}

AGRIANUAL. Anuário da agricultura brasileira. São Paulo: FNP Consultoria \& Agroinformativos, 2007. 504 p.

ARNY, D. C.; HANSON, E. W.; WORF, G. L.; OPLINGER, E. S.; HUGHES, W. H. Powdery mildew on soybean in Wisconsin. Plant Disease Reporter, Beltsville, v. 59, p. 288-290, 1975.

BEDENDO, I. P. Oídios. In: BERGAMIN FILHO, A.; KIMATI, H.; AMORIM, L. Manual de fitopatologia: princípios e conceitos. 3. ed. São Paulo: Agronômica Ceres, 1995. p. 866-871.

BRODIE, H. J.; NEUFELD, C. C. The development and structure of the conidia of Erysiphe polygoni DC and their germination at low humidity. Canadian Journal Research, Ontario, v. 20, p. 41-61, 1942.

BUZZELL, R. I.; HAAS, J. H. Powdery mildew of soybeans. Soybean Genetics Newsletter, Ames, v. 2, p. 7-9, 1975.

CAMPBELL, C. L.; MADDEN, L. V. Introduction to plant disease epidemiology. New York: Academic, 1990. 532 p.

DEMSKI, J. W.; PHILLIPS, D. V. Reactions of soybean cultivars to powdery mildew. Plant Disease Reporter, Beltsville, v. 58, p. 723-726, 1974.
DUNLEAVY, M. M. Soybean seed yield losses caused by powdery mildew. Crop Science, Madison, v. 18, p. 337-339, 1978.

GRAU, C. R.; LAURENCE, J. A. Observations on resistance and heritability of resistance to powdery mildew of soybean. Plant Disease Reporter, Beltsville, v. 59, p. 458-460, 1975.

JOHNSON, H. W.; PHILLIPS, D. V. Other legumes prove susceptible to a powdery mildew of Psoralea tenax. Plant Disease Reporter, Beltsville, v. 45, p. 542-543, 1961.

LEATH, S.; CARROLL, R. B. Powdery mildew on soybean in delaware. Plant Disease, Saint Paul, v. 66, p. 70-71, 1982.

LEITE, R. M. V. B. C.; AMORIM, L. Influência da temperatura e do molhamento foliar no monociclo da mancha de alternaria em girassol. Fitopatologia Brasileira, Brasília, v. 27, p. 193-200, 2002.

LOHNES, D. G.; BERNARD, R. L. Inheritance of resistance to powdery mildew in soybeans. Plant Disease, Saint Paul, v. 76, p. 964-965, 1992.

LOHNES, D. G.; NICKELL, C. D. Effects of Powdery Mildew Alleles Rmd-c, Rmd, and $r m d$ on yield and other characteristics in soybean. Plant Disease, Saint Paul, v. 78, p. 299-301, 1994.

MATTIAZZI, P. Efeito do oídio (Microsphaera diffusa Cooke e Peck) na produção e duração da área foliar sadia da soja. 2003. Tese (Doutorado em Fitotecnia) Escola Superior de Agricultura Luiz de Queiroz, Piracicaba, 2003.

MIGNUCCI, J. S. Powdery mildew. In: SINCLAIR, J. B.; BACKMAN, P. A. (Eds.). Compendium of soybean diseases. Saint Paul: APS, 1989. p. 21-23.

MIGNUCCI, J. S.; BOYER, J. S. Inhibition of photosynthesis and transpiration in soybean infected by Microsphaera diffusa Powdery mildew.

Phytopathology, Saint Paul, v. 69, p. 227-230, 1979.

MIGNUCCI, J. S.; CHAMBERLAIN, D. W. Interactions of Microsphaera diffusa with Soybeans and Other Legumes. Phytopathology, Saint Paul, v. 68, p. 169-173, 1978. 
MIGNUCCI, J. S.; LIM, S. M. Powdery mildew development on soybeans with adult plant resistance. Phytopathology, Saint Paul, v. 70, p. 919-921, 1980.

MIGNUCCI, J. S.; LIM, S. N.; HEPPERLY, P. R. Effects of temperature on reactions of soybean seedlings to powdery mildew. Plant Disease Reporter, Beltsville, v. 61, p. 122-124, 1977.

MIYASAKA, S.; MEDINA, J. C. A soja no Brasil. Campinas: ITAL, 1981. 1026 p.

PHILLIPS, D. V. Stability of Microsphaera diffusa and the effect of powdery mildew on yield of soybeans. Plant Disease, Saint Paul, v. 68, p. 953-956, 1984.

REIS, E. M. Previsão de doenças de plantas. Passo Fundo: UPF, 2004. 316 p.

REIS, E. M.; SARTORI, A. F.; CÂMARA, R. K. Modelo climático para previsão da ferrugem da soja. Summa Phytopathologica, Botucatu, v. 30, p. 290-292, 2004.

RITCHIE, S.; HANWAY, J. J.; THOMPSON, H. E. How a soybean plant develops. Ames: Iowa State University of Science and Technology, Cooperative Extension Service, 1982. 20 p. (Special report, 53).
SARTORATO, A.; YORINORI, J. T. Oídios de leguminosas: feijoeiro e soja. In: STANDNICK, M. J.; RIVERA, M. C. Oídios. Jaguariúna: Embrapa Meio Ambiente, 2001. p. 255-284.

TANAKA, M. A. S.; ITO, M. F.; MASCARENHAS, H. A. A.; DUDIENAS, C.; MIRANDA, M. A. C.

Desenvolvimento do oídio da soja em casa-devegetação. Summa Phytopathologica, Botucatu, v. 19, p. 125-126, 1993.

THOMAS, C. S.; GUBLER, W. D.; LEAVITT, G. Field testing of a powdery mildew disease forecast model on grapes in California. Phytopathology, Saint Paul, v. 84, p. 1070, 1994.

YORINORI, J. T.; CHARCHAR, M.; D’AVILA, J.; NASSER, L. C. B.; HENNING, A. A. Doenças da soja e seu controle. In: ARANTES, N. E.; SOUZA, P. I. M. Cultura da soja nos cerrados. Piracicaba: Potafos, 1993. p. 333-397.

YORINORI, J. T.; HIROMOTO, D. M. Determinação de perdas em soja causadas por doenças fúngicas. Londrina: Embrapa-CNPSo, 1998. (Documentos, 118). 